\title{
ASPECTOS SOBRE EL DEPÓSITO LEGAL DE PUBLICACIONES ESPAÑOLAS DE BELLAS ARTES ANDALUZAS (1987-1996)
}

\author{
José Luls Herrera Morillas*
}

Resumen: Después de localizar los documentos producidos en las ocho provincias andaluzas y en el resto de España, desde 1987 a 1996, que tienen por contenido las Bellas Artes y que hacen referencia a Andalucía, se realiza un estudio comparativo entre las obras localizadas y depositadas en la Biblioteca Nacional y la Biblioteca de Andalucía, distinguiendo las obras publicadas en cada año; las diferentes materias; las tipologías documentales y los lugares de publicación. Esta comparación y cuantificación de los datos permite también comprobar algunos aspectos relacionados con el cumplimiento del Depósito Legal en Andalucía. Éste se compara con el nacional y se aportan una serie de reflexiones y conclusiones al respecto.

Palabras clave: Depósito Legal, Bellas Artes andaluzas, bibliografía nacional, bibliografía regional, control bibliográfico nacional, control bibliográfico regional.

\begin{abstract}
A comparative study was carried out after locating documents produced between 1987 and 1996 in the eight provinces of Andalusia and in the rest of Spain. The documents refer to the area of Andalusia. Their subject-matter is that of the Fine Arts. The present study compares works localised and deposited in the National Library and in the Library of Andalusia and distinguishes among works by reference to year of publication, subject-matter, documental typology and place of publication. This comparison and quantification of the data also makes it possible to evaluate several aspects regarding the observance of the Legal Deposit in Andalusia. The latter is compared with the National Legal Deposit and a number of observations and conclusions are made.

Keywords: Legal Deposit, Andalusian Fine Arts, National Library, regional bibliography, national bibliographical control, regional bibliographical control.
\end{abstract}

\section{Introducción}

El propósito de este trabajo ha sido la localización de documentos (monografías y publicaciones periódicas) relacionados con las Bellas Artes -exceptuando el Cine, la Música y la Fotografía - de las obras publicadas o referidas a Andalucía, editadas en las ocho provincias andaluzas y en el resto de España, consiguiendo la exhaustividad dentro de los límites establecidos. A partir de este material he querido comprobar algunos aspectos relacionados con el cumplimiento del Depósito Legal en Andalucía, compararlo con el nacional y aportar algunas reflexiones al respecto.

* Profesor de E.P.O. Granada.

Recibido: 20-1-2000. 
La aparición desde los años 80 de unas bibliografías autonómicas ha hecho surgir una polémica sobre la justificación y utilidad de éstas, que se centra en la duplicación de contenido y de tareas que implica su existencia junto a la bibliografía nacional. Parte de esta investigación y de las conclusiones que obtenga a partir de ella van a estar en relación con lo que plantea Cordón García (1), cuando afirma:

«lo que sería interesante comprobar es qué parte de la bibliografía andaluza aparece publicada en Bibliografía Española, con objeto de conocer el grado de duplicidad que se produce, y al tiempo comprobar si el control bibliográfico ejercido desde la Comunidad mejora los resultados del control efectuado en el ámbito nacional» .

El estudio comprende monografías y publicaciones periódicas publicadas en España desde 1987 a 1996. El primero es el año en que la Biblioteca de Andalucía empieza a recibir el Depósito Legal. El año 1996 cierra el periodo de estudio, porque una vez que empecé a disponer de las cifras de obras publicadas por años, observé que las publicaciones surgidas en esta década eran suficientes para realizar la investigación. Además, las obras publicadas con posterioridad a 1996, en la fecha que decidí terminar la recogida del material para su estudio, enero de 1998 , no estaban en su totalidad disponibles en las bibliotecas consultadas. También he seleccionado obras que están relacionadas con las Bellas Artes pero que pueden incluirse dentro de otras materias. Es el caso de obras de Arqueología, de guías turísticas y de publicaciones de carácter descriptivo o geográfico referentes a ciudades andaluzas. En este caso he incluido sólo las que pueden tener más relación e interés.

Para localizar las obras he utilizado el Boletín del Depósito Legal de Andalucía serie A «Monografías»; el Boletín del Depósito Legal de Andalucía serie B «Publicaciones Periódicas» (hasta 1992 el Boletín que recogía las monografías y el que incluía las publicaciones periódicas fueron publicados en formatos independientes, desde 1993 aparecen en el mismo volumen físicamente juntos); Bibliografía Española, desde 1987 a 1998 (en papel y en CD-ROM) y las bases de datos Ariadna y Tartesos.

En total he localizado 2.116 obras (2.031 monografias y 85 publicaciones periódicas). Están incluidas las publicaciones que se encuentran ubicadas a la vez en las dos bibliotecas y también las obras que sólo he localizado en la Biblioteca Nacional o en la Biblioteca de Andalucía. Estas cifras son de la última búsqueda global realizada en julio de 1998. Con anterioridad realizamos otra búsqueda global (julio de 1997) con los siguientes resultados: Total monografías: 1.960; duplicadas: 1.081; sólo en Biblioteca Nacional: 374; sólo en Biblioteca de Andalucía: 505. Los datos de las publicaciones periódicas coinciden en las dos búsquedas.

Como se observa, los datos más interesantes son los de las monografías. Dentro de éstas, las obras duplicadas son 1268; las que sólo están en la Biblioteca Nacional, 332 y las que sólo están en la Biblioteca de Andalucía, 431. Con estos dos últimos resultados he comprobado que el Depósito Legal y la obligación que tiene la Biblioteca de Andalucía de adquirir todo lo publicado sobre Andalucía fuera de este lugar (que no lo ingresa por Depósito Legal y la Biblioteca Nacional sí) no se cumple en su totalidad. También, estos resultados demuestran que gran parte de los ejemplares que se recogen a través del Depósito Legal se encuentran en las dos Bibliotecas y, como consecuencia, esta duplicación se traslada a las bibliografías, elaboradas a par- 
tir de las obras ingresadas por el Depósito Legal, por cada una de las Bibliotecas citadas.

Con estos datos la investigación realizada ha ido más lejos de la comprobación sugerida por José Antonio Cordón - citada al principio del artículo- que consistía en ver la parte de la bibliografía andaluza que aparece publicada en Bibliografía Española: pues he utilizado como fuente no sólo las bibliografías impresas sino también las bases de datos de ambas Bibliotecas, y como he detectado que no todas las obras que recogen estas bases de datos aparecen en las bibliografías andaluza y nacional, tenemos que el número de obras recopiladas y por lo tanto cotejadas entre ambas bibliotecas ha sido mayor.

Esta realidad, que se da hoy en día en nuestro país, de la aparición simultánea - pero con una elaboración independiente- de la bibliografía nacional y las bibliografías regionales hace dudar de la necesidad y justificación de estas bibliografías regionales. El origen de esta problemática hay que buscarlo en la descentralización surgida tras la vertebración del Estado en comunidades autónomas que va a influir en el desarrollo del control bibliográfico. Las distintas comunidades reciben competencias en materia de cultura y subsidiariamente en la gestión del Depósito Legal, lo cual abre nuevos canales para el control bibliográfico en la medida en que la unidad geográfica sobre la que se ejerce es menor. Por otra parte, la creación de bibliotecas regionales ha incrementado el seguimiento de las publicaciones autóctonas, al tiempo que las difunde mediante la elaboración de bibliografías regionales.

Para Carrión Gútiez (2) «de una bibliografía nacional pueden salir muchas bibliografías autonómicas, de muchas bibliografías autonómicas no puede surgir una bibliografía nacional». Es decir, las bibliografías regionales no son necesarias por razones económicas y prácticas.

De Torres Ramírez (3), después de reconocer la duplicación de trabajo que supone la existencia de ambas bibliografías, justifica la existencia de las bibliografías regionales siempre que amplíen la cobertura de documentos recopilados.

La problemática planteada es una cuestión polémica, que es pronto para ver cerrada debido a que la aparición de estas bibliografías es todavía un hecho reciente (Cataluña 1983, Galicia 1980, Asturias 1987, País Valenciano y Andalucía 1988) y que tiene su origen en una realidad, que me parece sin duda muy positiva, el establecimiento de las bibliotecas regionales que lleva a una descentralización de los fondos, respecto a Madrid.

\section{Análisis de los datos: monografías}

De las obras recogidas en este estudio la Biblioteca Nacional tiene en total 1600 (de las cuales 332 no están en la andaluza) y la Biblioteca de Andalucía 1699 (de las cuales 432 no se encuentran en Madrid). A la primera le faltan 432 obras y a la segunda 332 [estos datos son de la búsqueda de 1998. Los de 1997 son: total obras Biblioteca Nacional: 1.455; total obras Biblioteca de Andalucía: 1.586].

La Biblioteca Nacional debería tener todo lo que guarda la Biblioteca de Andalucía pero vemos que no se cumple, al menos en el campo temático investigado. En el comentario sucesivo que voy a ir realizando de los datos obtenidos, tendré ocasión de exponer posibles causas de esta falta de coincidencia entre la teoría y la práctica. Tan 
sólo voy a adelantar una cuestión: este estudio ha tenido como fuentes las bibliografías impresas y las bases de datos elaboradas por las dos bibliotecas y no existe correspondencia exacta entre estos instrumentos de búsqueda y el Depósito Legal, realidad de sobra conocida y que está presente en todas las partes del mundo. Según el Congreso de París de 1977 debe ser la agencia bibliográfica correspondiente (en nuestro caso las respectivas bibliotecas) la que decide lo que se debe incluir o no del Depósito Legal en los productos bibliográficos elaborados. La diferencia que he detectado entre los fondos de una y otra biblioteca se puede deber a diferentes criterios de selección.

Si comparamos las cifras totales de una y otra biblioteca (figura 1) observamos que hasta 1991 la Biblioteca de Andalucía tiene más fondos sobre Arte referidos a Andalucía que la Nacional, pero en el período 1992-1995 la tendencia cambia para luego recuperarse en 1996.

Figura 1

Monografías de Bellas Artes

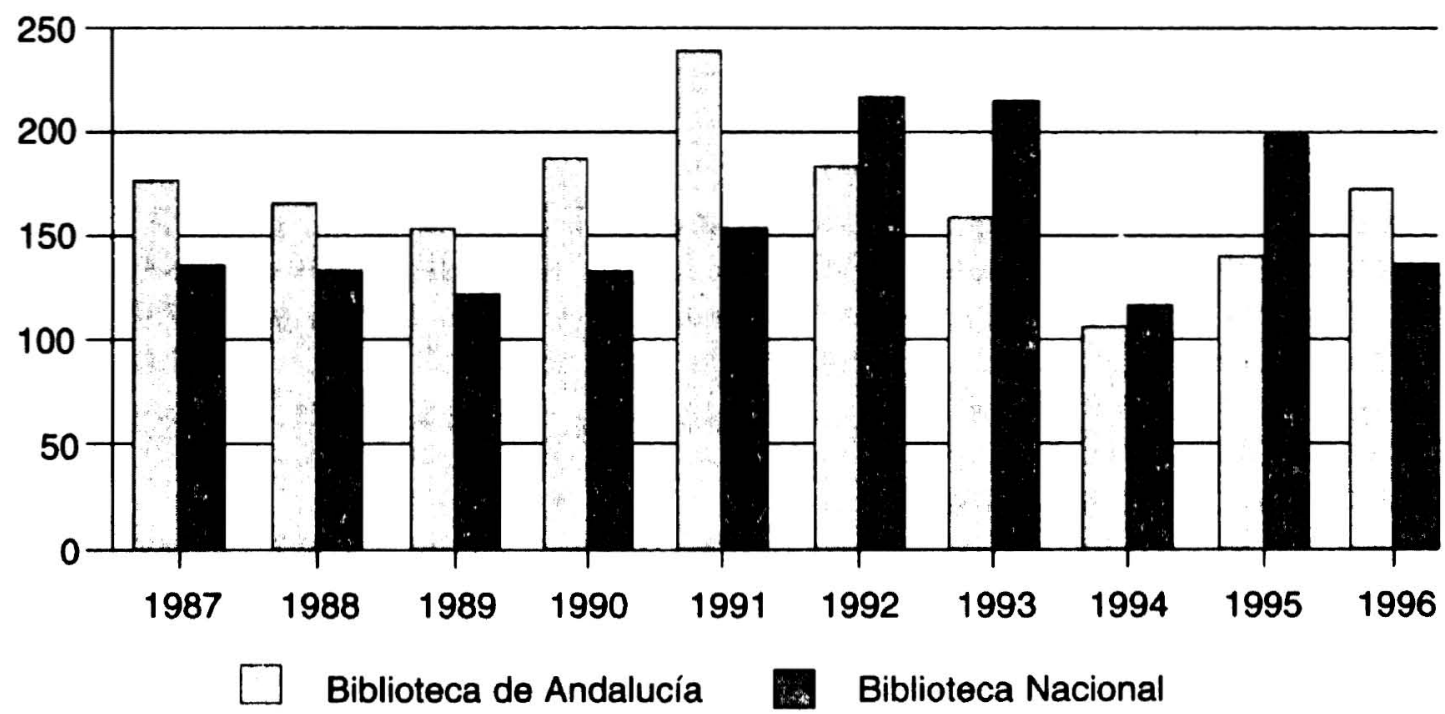

La evolución observada hasta 1991 coincide con los datos del estudio realizado por Cordón García (4) para comprobar la parte de la bibliografía andaluza que se repite en Bibliografía Española en las obras registradas en el año 1991 (en este trabajo utiliza como fuente los datos de ingresos que figuran en la memoria del Depósito Legal del año 1991 publicada por la Biblioteca de Andalucía y también realiza una búsqueda en el CD-ROM de Bibliografía Española). A continuación recojo su interpretación sobre el hecho de que las cifras de la Biblioteca Andaluza sean superiores:

«La mejora de los resultados pensamos que se debe a una acertada gestión y al hecho de que, desde el año 1990, se automatiza el proceso de descripción de registros relativos al Depósito Legal». 
Este mismo autor también compara la cuantificación efectuada por la Biblioteca Nacional y la Biblioteca de Andalucía de las obra ingresadas por Depósito Legal en las provincias andaluzas en los años 1991 y 1993. En el primer año, la de Andalucía está a la cabeza y, en el segundo, la Nacional. Sobre los resultados de 1991 da las siguiente explicación:

«En principio podríamos atribuir la diferencia al procedimiento estipulado para la entrega de ejemplares, que recae sobre las oficinas provinciales, las cuales han de remitir, de todos los materiales estipulados por la ley, los ejemplares correspondientes a la Biblioteca de Andalucía y los relativos a la $\mathrm{Bi}$ blioteca Nacional, de tal manera que pueden no mostrar la misma diligencia en el cumplimiento con Madrid que con la biblioteca de la comunidad».

Respecto al cambio de tendencia observado en 1993 establece esta justificación:

«la Biblioteca de Andalucía se vio sometida a un proceso de reformas y traslados que concluyeron con su instalación definitiva en la ubicación que hoy tiene».

Otra causa para justificar las diferencias entre la Biblioteca Nacional y la andaluza (además de la expuesta arriba sobre la actuación de las oficinas provinciales) puede deberse a las obras que una y otra biblioteca no incluyen a la hora de catalogar los documentos recibidos por Depósito Legal y que luego pasan a formar parte de los fondos de las respectivas bibliotecas y del contenido de las bibliografías impresas elaboradas. Después de realizar la segunda búsqueda definitiva en 1998 y comparar los resultados con los obtenidos en la búsqueda de 1997 he observado que las diferencias entre una y otra biblioteca disminuyen; por lo tanto, la ausencia de obras también se debe a la diferencia de tiempo empleado por estos centros bibliotecarios para poner disponibles las obras que ingresan.

También han podido influir las dificultades de funcionamiento por las que ha pasado la Biblioteca de Andalucía desde su traslado al edificio actual, que he podido constatar en las visitas realizadas durante mi trabajo. Señalo algunas: cambios en la dirección de este centro; carencia de personal para mantener un horario amplio de consulta; modificación de criterio a la hora de la distribución de los fondos en la biblioteca; pérdidas de libros; signaturas que no corresponden con los ejemplares o que remiten a obras que no están en su lugar y obras registradas en la base de datos que carecen de signatura. Por contraste, puedo afirmar que todas las obras que seleccioné para consultar en la Biblioteca Nacional estaban depositadas y disponibles.

El año con más obras en la Biblioteca Nacional es 1992 (219 obras); le siguen por orden de obras ingresadas: 1993 (217 obras), 1995 (198 obras), 1991 (154 obras), 1996 (138 obras), 1987 (136 obras), 1990 (134 obras), 1988 (133 obras), 1989 (124 obras) y 1994 (117 obras).

En la Biblioteca de Andalucía en primer lugar está 1991 (242 obras) y le siguen: 1990 (189 obras), 1992 (184 obras), 1987 (177 obras), 1996 (173 obras), 1988 (165 obras), 1993 (160 obras), 1989 (154 obras), 1995 (142 obras), y 1994 (108 obras).

Seguidamente nos vamos a centrar en el análisis de las obras que sólo hemos localizado en cada biblioteca y que no están en las dos, es decir que no están duplica- 
das, como es lo esperado, y como de hecho sucede con la mayoría de las obras localizadas. Estas obras son las más interesantes desde el punto de vista comparativo pues es lo que diferencia a los dos centros.

\subsection{Estudio y comparación de las obras no duplicadas, localizadas sólo en la Biblioteca Nacional o en la Biblioteca de Andalucía}

\subsubsection{Comparación de los datos globales de cada biblioteca: número de obras publicadas por años, por materias y por tipologías documentales}

De las 2.031 obras recogidas, 332 [374] sólo se encuentran en la Biblioteca Nacional y 431 [505] sólo en la Biblioteca de Andalucía. Voy a colocar junto a los datos de la última búsqueda global de las obras, realizada en 1998, los datos de 1997 (búsqueda anterior). Irán entre corchetes y en cursiva, junto a los primeros. Cuando no aparezcan datos en cursiva es que coinciden con los anteriores.

Al observar la distribución de estas obras por años (figura 2), desde 1987 hasta 1991 la Biblioteca de Andalucía tiene más obras que la Biblioteca Nacional, tendencia que se invierte en el período 1992-1995. Es la misma evolución que se aprecia al comparar las obras totales de la Biblioteca Nacional y las de la Biblioteca de Andalucía, realizada al inicio del estudio.

El año con más obras, en la Biblioteca de Andalucía, es 1991 [101] [108] y con menos 1994 [11] [13]. En la Biblioteca Nacional es 1995 [71] [75] y 1990 [11] respectivamente. Dentro de la distribución por materias (figura 3) las obras más nume-

Figura 2

Comparación: obras totales de la Biblioteca Nacional y Biblioteca de Andalucía

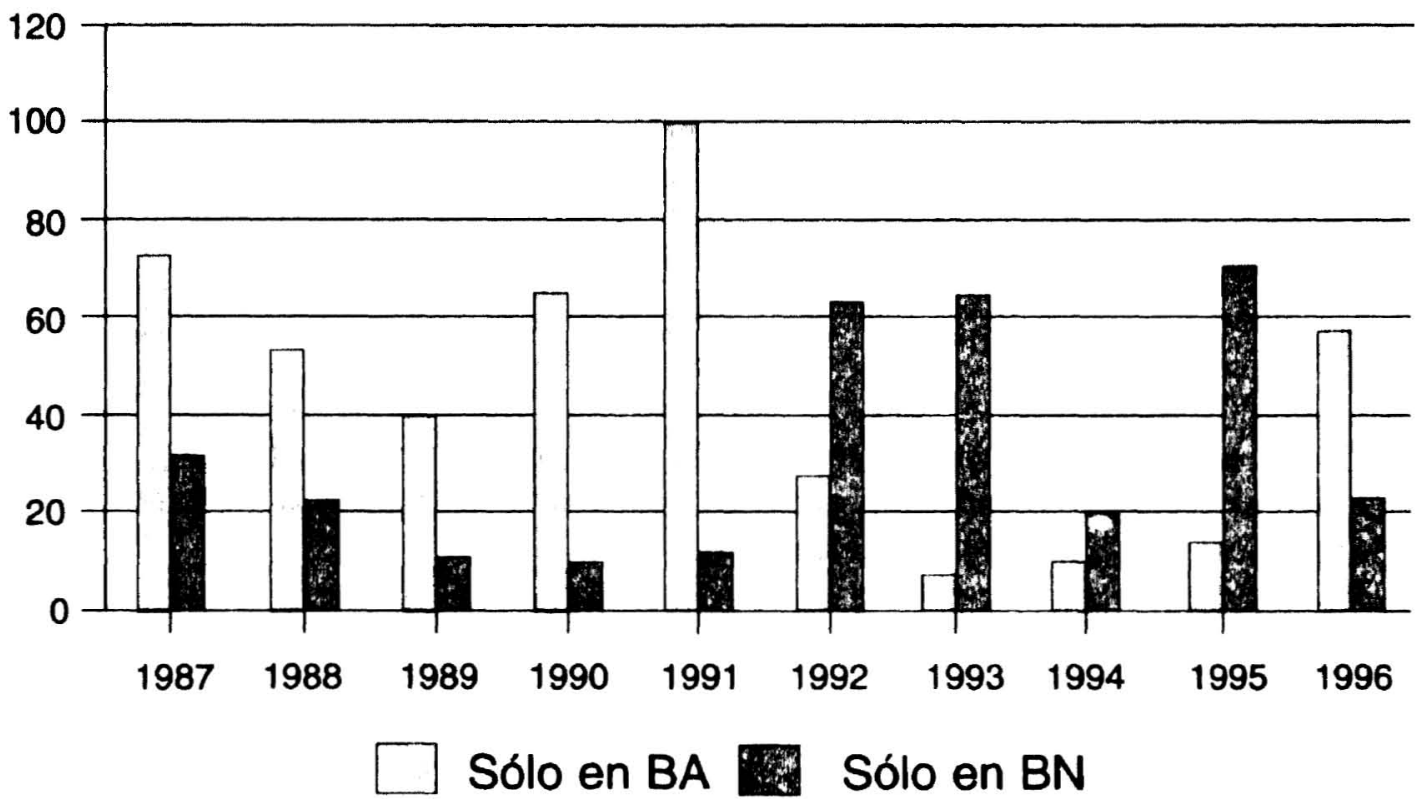


Figura 3

Comparación: materias Biblioteca Nacional, Biblioteca de Andalucía

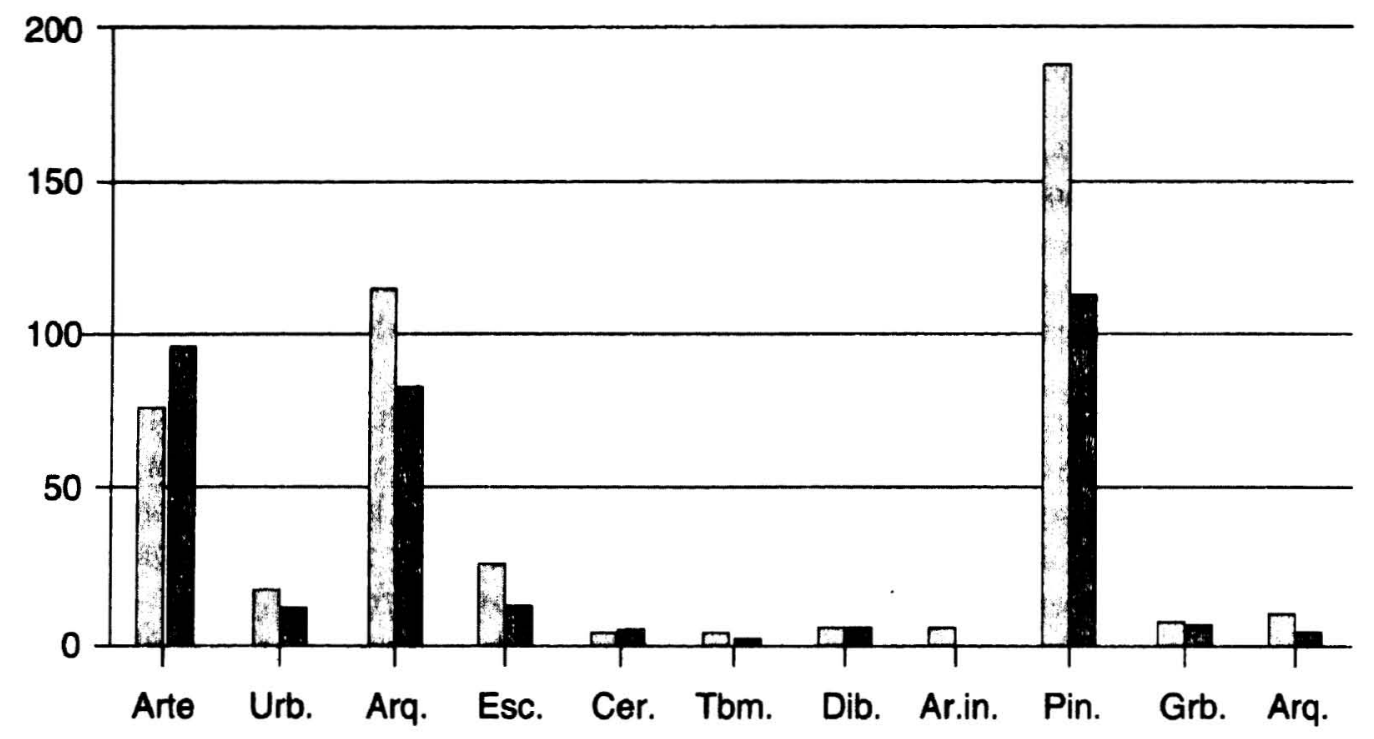

Sólo en BA Sólo en BN

rosas, en las dos bibliotecas, son las correspondientes a Pintura, Arte en general y Arquitectura. De Arte en general hay más obras en la Biblioteca Nacional; de Pintura y Arquitectura en la Biblioteca de Andalucía.

Respecto a las tipologías documentales (figura 4), en la Biblioteca Nacional siguen este orden:

1. ${ }^{\circ}$ Libros: 230 [268].

2. Catálogos de exposiciones: 136 [149].

3. Folletos: 103 [106].

4. ${ }^{\circ}$ Guías: 36 [39].

5. Tesis: 9 [11].

6. ${ }^{\circ}$ Obras pedagógicas: 6 [7].

7. ${ }^{\circ}$ Otros: 2 [4].

De los catálogos de exposiciones 66 [76] son libros y 71 [73] folletos. Las obras pedagógicas todas están formadas por folletos. Dentro del apartado otros se incluyen: 2 Homenajes, [1 Congreso] y 1 Encuentro.

En la Biblioteca de Andalucía:

1. ${ }^{\circ}$ Folletos: 267 [281].

2. Catálogos de exposiciones: 208 [230].

3. ${ }^{\circ}$ Libros: 167 [227].

4. ${ }^{\circ}$ Obras pedagógicas: 112 [116]. 
5. ${ }^{\circ}$ Guías: 8 [14].

6. ${ }^{\circ}$ Otros: 5 [11].

7. Tesis: 3 [4].

De los catálogos de exposiciones 150 [160] son folletos y 58 [70] libros. Dentro de las obras pedagógicas se incluyen 82 [83] folletos y 30 [33] libros. El apartado otros reúne: 1 [5] Congreso, 3 Jornadas, 2 Homenajes y 1 Encuentro.

La Biblioteca de Andalucía recoge más folletos, catálogos de exposiciones y publicaciones de los gabinetes pedagógicos que la Biblioteca Nacional. Por el contrario la Biblioteca Nacional supera a la andaluza en libros, tesis y guías. Si tenemos en cuenta que la mayoría de los catálogos de exposiciones y obras pedagógicas de la $\mathrm{Bi}$ blioteca de Andalucía son folletos (mientras que en la Biblioteca Nacional son libros) está claro que la diferencia entre los fondos de una y otra Biblioteca se encuentra principalmente en los folletos.

Este resultado sirve para comprobar la validez del planteamiento de Isabel de Torres - ya citado con anterioridad - según el cual las bibliografías regionales estarían justificadas siempre que amplíen la cobertura de los materiales, incluyendo aquéllos que no guarda la Biblioteca Nacional.

Otra explicación a la diferencia que existe entre los folletos registrados por las dos bibliotecas puede estar en el hecho siguiente: la Biblioteca Nacional, debido a la cantidad de obras que recibe, al catalogarlas suele no tener en cuenta algunas. Actúa de manera selectiva sobre el conjunto de materias que son objeto de Depósito Legal. Este mismo criterio se sigue a la hora de elaborar Bibliografía Española, como señala Girón García (5): «la cobertura de Bibliografía Española coincide con los materiales que

Figura 4

Comparación: Tipologías documentales, Biblioteca Nacional y Biblioteca de Andalucía

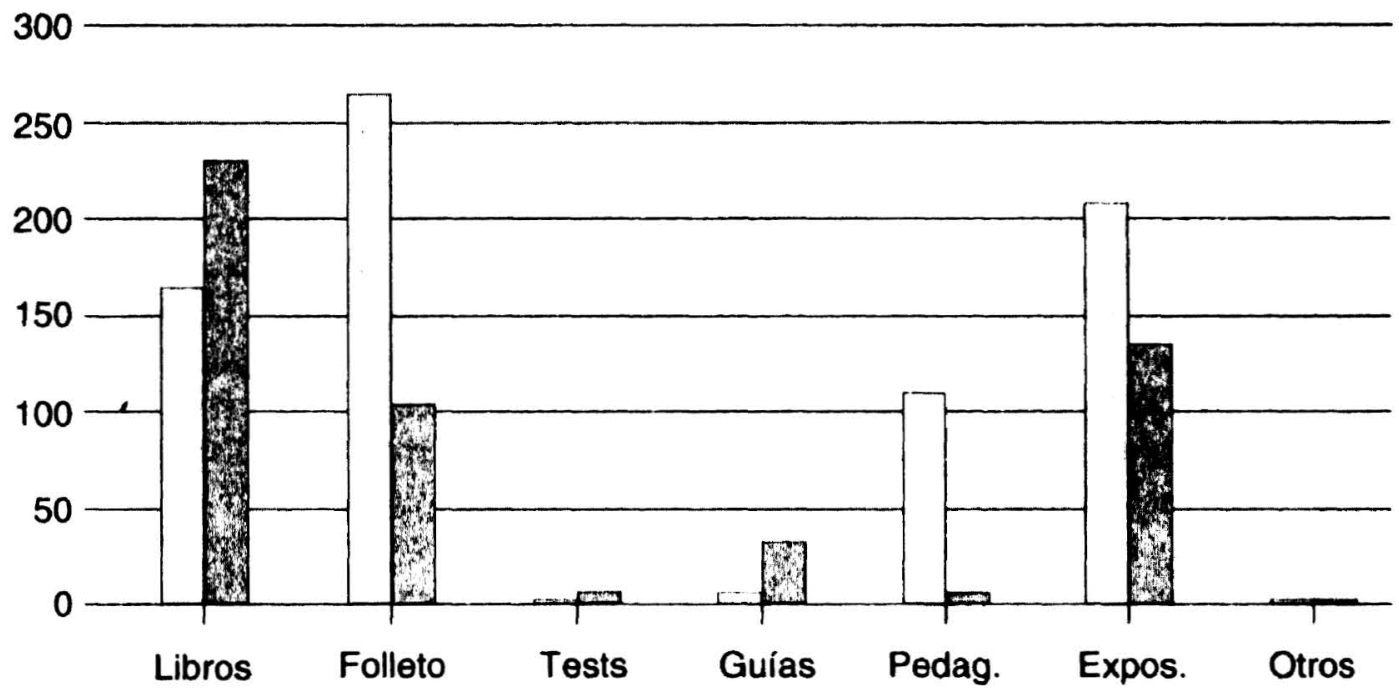

Sólo en BA Sólo en BN 
se catalogan para su inclusión en el catálogo general de la Biblioteca Nacional». Es lógico que entre estas obras se encuentren algunos folletos, que por su poca consistencia no se recogen en favor de otras obras de mayor envergadura. Fuentes Romero (6), sobre esta problemática, ha escrito lo siguiente:

«Incorporar todo este material al fondo de los demás libros es algo que no vemos ni viable ni práctico, y ello por varias razones. Esta ingente cantidad de material tendrá, con el paso del tiempo, un valor, al menos cultural, enorme; pero en el momento en que aparece son mínimas [...]. Además está el hecho de que la mayoría de las bibliotecas no andan tan sobradas de espacio como para ocuparlo con un material que, hoy por hoy, es, como ya hemos dicho, de poco manejo...».

La Biblioteca de Andalucía todavía no ha iniciado esta estrategia, aunque se la está planteando. De ponerse en práctica, muchos de estos folletos tendrían el mismo destino que hoy tienen otros materiales como las postales, carteles, etc., que se conservan y guardan en los depósitos de la biblioteca pero no se incluyen en las bibliografías elaboradas, ni en las bases de datos puestas al servicio de los usuarios.

La Biblioteca Nacional tiene un mayor número de guías que la andaluza. Muchas de éstas guías se publican en Madrid y Barcelona, llegan a Madrid por Depósito Legal. La Biblioteca de Andalucía debería conseguirlas, al ser obras de tema andaluz. Lo mismo ocurre con las tesis doctorales, entre las que se encuentran las publicadas por la Universidad Complutense, ausentes de los fondos de la Biblioteca de Andalucía.

\subsubsection{Estudio de las obras sólo localizadas en la Biblioteca Nacional}

\subsubsection{Características de las obras publicadas en Madrid, Barcelona y Andalucía}

Madrid: 91 [99].

Barcelona: 47.

Almería: 4 [5].

Cádiz: 3 [6].

Córdoba: 23 [30].

Granada: 32 [35].

Huelva: 2 [5].

Jaén: 17 [19].

Málaga: 29 [33].

Sevilla: 43 [51].

Otras ciudades: 28 [29].

S.l.: 1 [2].

Las obras publicadas fuera de Andalucía, 175 [166], aunque la Biblioteca andaluza no las reciba por Depósito Legal tiene el deber de adquirirlas. Al no hacerlo, según la ley de Bibliotecas para Andalucía de 1983, se incumple una de las funciones que señala: «recoger, conservar y difundir toda la producción impresa, sonora y visual de Andalucía o sobre Andalucía». 
Según Cordón García (4) éste es un grave problema con el que se encuentran estas instituciones para conseguir el control bibliográfico que les marca la ley pues, siguiendo a Beaudiquez, estaríamos ante la elaboración de una bibliografía documental. Es decir cumplir al pie de la letra esta ley supondría: recoger la producción de los autores andaluces allá donde éstos publiquen, recoger las obras sobre cualquier aspecto geográfico, político, cultural, económico de tierras andaluzas, independientemente de donde esto se produzca. Esto, desde el punto de vista del control bibliográfico, no deja de ser una formulación curiosa pero irrealizable.

Las demás obras, por estar publicadas en las provincias andaluzas, lo lógico es que estuvieran también en la Biblioteca de Andalucía y que hubieran ingresado por Depósito Legal. Esta deficiencia puede explicarse en unos casos por errores en la recepción del mismo o por retraso en la catalogación de las obras.

A continuación voy a estudiar estas publicaciones agrupándolas en: obras publicadas en Madrid, obras publicadas en Barcelona y obras publicadas en Andalucía. De cada grupo comentaré las materias y tipologías documentales.

Las materias de las obras publicadas en Madrid están formadas por:

- Arte: 28 [30] obras. Abundan las guías turísticas de ciudades, 9 [10], guías de museos y de monumentos, 7 y monografías sobre diferentes períodos del arte andaluz: 7.

- Urbanismo: 2 [3] obras sobre jardines [y 1 estudio sobre historia del urbanismo árabe en Andalucía].

- Arquitectura: 29 [32] obras. Las obras más numerosas están dedicadas a gú́as divulgativas para visitar monumentos: 15 [18] -especialmente la Alhambra de Granada, la Mezquita de Córdoba y la Catedral de Sevilla-; trabajos de investigación: 7 y catálogos de exposiciones: 2 .

- Escultura: 3. Dos obras de investigación y una obra de carácter divulgativo sobre escultura barroca.

- Cerámica artística: 4 obras. Están dedicadas al estudio de colecciones y del material cerámico procedente de yacimientos arqueológicos.

- Trabajos artísticos en metal: 2 obras. 1 tesis doctoral y 1 catálogo de exposición de piezas religiosas.

- Dibujo: 1 catálogo de exposición.

- Artes industriales: 1 catálogo de exposición.

- Pintura: 24 [25]. Catálogos de exposiciones: 10 [11], sobre pintura contemporánea: 6 [7], sobre pintura histórica: 4; de todas estas exposiciones 6 han tenido lugar en Madrid y 5 en Andalucía (en estos casos no coincide el lugar de publicación del catálogo con el lugar en el que se ha celebrado la exposición). Obras divulgativas sobre pintores famosos, como por ejemplo Murillo: 9 y trabajos de investigación: 4 .

- Grabados: 0.

- Arqueología: 0.

Las tipologías documentales de las obras publicadas en Madrid incluyen:

- Libros: 114 [121].

- Folletos: 18. 
- Tesis: 8.

- Guías: 13 [14.]

- Obras pedagógicas: 0.

- Catálogos de exposiciones: 17 [18]. 9 [10] son libros y 8 folletos.

En las materias de las obras publicadas en Barcelona están representados:

- Arte: 24 obras. 21 son guías turísticas de ciudades.

- Urbanismo: 1 obra sobre parques y jardines.

- Arquitectura: 19 obras. 17 son guías de monumentos, 5 están dedicadas a la Mezquita de Córdoba y 4 a la Alhambra de Granada.

- Pintura: 3 obras de divulgación sobre pintores famosos, 2 están dedicadas a Velázquez y 1 a Murillo.

Del resto de las materias no se ha publicado ninguna obra.

Las tipologias documentales de las obras publicadas en Barcelona contienen:

- Libros: 45.

- Folletos: 6.

- Tesis: 0.

- Guías: 20.

- Obras pedagógicas: 0.

- Catálogos de exposiciones: 1.

Las materias de las obras publicadas en Andalucía están representadas por:

- Arte: 38 [45] obras. Catálogos de exposiciones: 17, 16 de pintura contemporánea y 3 de pintura histórica. Publicaciones sobre arte en general: 12 [14] y guías turísticas de ciudades: 4 [6].

- Urbanismo: 8 [9] obras. Trabajos de investigación: 5 [6], sobre jardines: 1 y de carácter divulgativo: 1 .

- Arquitectura: 30 [38] obras. De carácter divulgativo sobre edificios y monumentos: 12 [13]; trabajos de investigación: 8 [13]; sobre arquitectura en general 8 y catálogos de exposiciones: 1 [2].

- Escultura: 9 [14] obras. Catálogos de exposiciones sobre escultura contemporánea: 8 [10] y obras sobre imaginería: 2 [4].

- Cerámica artística: 2 obras, 1 estudio de las piezas de una colección y 1 catálogo de exposición.

- Trabajos artísticos en metal: 1 obra sobre orfebrería religiosa.

- Dibujo: 6 obras. Casi todas catálogos de exposiciones [5].

- Artes industriales: 4 obras, 3 catálogos de exposiciones y 1 trabajo de investigación.

- Pintura: 71 [83] obras. Catálogos de exposiciones 69 [78]; 64 [72] de pintura contemporánea y 4 [5] de pintura histórica. Libros de pintura en general: 3 [y trabajos de investigación: 2].

- Grabados: 3 obras, 2 catálogos de exposiciones y 1 estudio de una colección. 
- Arqueología: 3 [5] obras. De carácter divulgativo: 3 y sobre los fondos de museos: 1 [2].

Las tipologías documentales de las obras publicadas en Andalucía incluyen:

- Libros: 79 [109].

- Folletos: 64 [67].

- Tesis: 3.

- Guías: 2.

- Obras pedagogicas: 6 [7].

- Catálogos de exposiciones: 97 [106].

\subsubsection{Estudio de las obras sólo localizadas en la Biblioteca de Andalucía}

\subsubsection{Características de las obras publicadas en Madrid, Barcelona y Andalucía}

El número de obras publicadas por provincias es el siguiente:

Madrid: 4 [5].

Barcelona: 2.

Almería: 12 [13].

Cádiz: 24 [30].

Córdoba: 60 [70].

Granada: 75 [91].

Huelva: 12 [14].

Jaén: 37 [39].

Málaga: 66 [76].

Sevilla: 59 [85].

Las materias de las obras publicadas en Madrid están representadas por:

- Arte: [1 obra].

- Pintura: 3 obras. Catálogos de exposiciones.

- Artes industriales: 1 obra.

Las tipologías documentales de las obras publicadas en Madrid contienen:

- Libros: 1 [2].

- Folletos: 3.

- Catálogos de exposiciones: 4. De ellos 3 son folletos y 1 es libro.

- Guías: [1].

Las obras publicadas en Barcelona están formadas por 2 libros de Arquitectura, uno sobre viviendas y castillos de Andalucía y otro sobre palacios de Al-Andalus. Recogen estudios de especialistas en la materia y abundantes ilustraciones. Constitu- 
yen dos cuidadas publicaciones de la editorial Lumwerg, vinculada al «Legado Andalusí».

Estos libros no han entrado en la biblioteca por Depósito Legal, quizás por compra o donación, pero la cifra es muy baja en comparación con los obras que se han publicado fuera de Andalucía. La Biblioteca de esta Comunidad debería prestar más atención a la localización y adquisición de estas obras. Tarea que puede incumplir por falta de presupuesto, necesario para la compra de obras, o por no estar incluidas dentro del plan de trabajo.

Las materias de las obras publicadas en Andalucía están representadas por:

- Arte: 69 [77] obras. 30 [31] publicaciones pedagógicas y de divulgación publicadas por los diferentes gabinetes pedagógicos de las capitales andaluzas con la finalidad de facilitar el conocimiento entre los escolares del patrimonio artístico de cada provincia. 26 catálogos de exposiciones. El resto de las obras son guías turísticas, de monumentos o museos; publicaciones de congresos y otros libros de arte en general.

- Urbanismo: 20 [21] obras. 7 [8] obras de investigación sobre aspectos urbanísticos de Andalucía, en varios casos desde una perspectiva histórica. 6 publicaciones de los gabinetes pedagógicos. 3 sobre inventario y descripción de conjuntos o cascos históricos. El resto de las obras tratan de congresos, planificación, concursos y proyectos.

- Arquitectura: 106 [118] obras. 73 publicaciones de los gabinetes pedagógicos. 13 [16] de investigación. 16 sobre arquitectura en general. 4 guías. El resto: directorios de arquitectos, exposiciones, restauración, concursos, inventarios y fuentes documentales.

- Escultura: 15 [20] obras. 14 [16] catálogos de exposiciones (todos, menos uno, son de escultura contemporánea), [2] obras de escultura religiosa, 1 obra de carácter pedagógico y divulgativo y otra que recoge documentos.

- Cerámica artística: 6 obras. 4 catálogos de exposiciones y las demás sobre piezas de colecciones.

- Trabajos artísticos en metal: 2 catálogos de exposiciones sobre orfebrería.

- Dibujo: 7 obras. 6 catálogos de exposiciones y 1 monografía sobre la producción de un artista.

- Artes industriales: 5 obras. 3 de carácter divulgativo y pedagógico, 1 catálogo de exposición y un folleto de una escuela taller.

- Pintura: 145 [157] obras. 131 [136] catálogos de exposiciones, prácticamente la totalidad de pintura contemporánea; el resto de las obras son de investigación - entre ellas alguna tesis-, de historia de la pintura y otras de carácter pedagógico y divulgativo.

- Grabados: 7 obras. 5 catálogos de exposiciones. 2 obras sobre colecciones de grabados.

- Arqueología: 8 [10] obras. 6 [8] de carácter divulgativo y pedagógico, casi todas sobre visitas a museos arqueológicos o sobre sus fondos; las 2 restantes estudian piezas de Arqueología.

Las tipologías documentales de las obras publicadas en Andalucía son: 
- Libros: 89 [148].

- Folletos: 229 [243].

- Tesis: 3 [4].

- Guias: [4].

- Obras pedagógicas: 111 [115]. De éstas 21 [24] son libros y 90 [91] folletos.

- Catálogos de exposiciones: 179 [201]. Formados por 35 [47] libros y 140 [154] folletos; 174 [194] tienen como contenido exposiciones de arte contemporáneo y 5 [7] arte de épocas anteriores.

- Otros: 16 [22] obras. Incluyen certámenes, muestras y homenajes.

La cobertura de la Biblioteca de Andalucía es mayor en folletos y dentro de éstos en catálogos de exposiciones y publicaciones de los gabinetes pedagógicos de Bellas Artes.

\section{Análisis de los datos: publicaciones periódicas}

Las publicaciones periódicas seleccionadas sobre Bellas Artes de Andalucía aparecidas entre los años 1987 y 1996 han sido 85 (de éstas se recoge sólo el n. ${ }^{\circ}$ 1, es decir sólo cuando salen por primera vez y también si cambian de título). De estas obras 48 están en las dos bibliotecas, Nacional y andaluza. Mientras que 15 sólo las he localizado en la Biblioteca Nacional y 22 en la Biblioteca de Andalucía. El número total de obras recogidas en la primera biblioteca ha sido 63 y en la segunda 70 . La situación es similar a la comprobada en el estudio de las monografías (y por tanto las causas que la explican): por un lado no coincide el número de obras de Bellas Artes referidas a Andalucía en las dos bibliotecas y por otro lado la Biblioteca de Andalucía alcanza una cifra mayor en las obras recopiladas.

\subsection{Publicaciones periódicas sobre Bellas Artes andaluzas, localizadas sólo en la Biblioteca Nacional o en la Biblioteca de Andalucía}

Una vez analizadas por separado las publicaciones periódicas que únicamente se localizan en la Biblioteca Nacional [15] y por otro lado, las que sólo están en la Biblioteca de Andalucía [22] he comprobado que no existe ninguna peculiaridad en la materia, tipología, año o lugar de publicación que caracterice a las obras de cada biblioteca y que, de haberla, podría servir de pista para explicar el porqué no se da la obligada doble ubicación de estas obras. Sirva de anécdota significativa que la única publicación periódica, de las localizadas, publicada fuera de Andalucía (en Madrid) no está en la Biblioteca andaluza; y es misión del citado centro bibliotecario la adquisición de todo lo publicado sobre Andalucía. Vemos con un nuevo ejemplo cómo esta finalidad no se cumple del todo.

Por tanto, de estas 15 obras de la Biblioteca Nacional, 14 están publicadas en ciudades andaluzas y sin embargo no están en la Biblioteca central de esta Comunidad Autónoma.

También queda reflejado con estos datos que existen obras ingresadas por Depó- 
sito Legal que aparecen en la Biblioteca Nacional y no en la andaluza y viceversa. En unos casos se debe a omisiones a la hora de hacer llegar, por parte de las oficinas provinciales, los ejemplares correspondientes de las obras ingresadas por Depósito Legal, unas veces a Granada y otras a Madrid. Cuando lo correcto es que de cada obra ingresada en cualquier provincia andaluza se hagan llegar los ejemplares correspondientes a los dos centros indicados. Y en otros casos, la diferencia se debe al tiempo empleado por una y otra biblioteca en la catalogación de las publicaciones.

\section{Conclusiones}

Las publicaciones periódicas que han aparecido en estos diez años alcanzan unos valores bajos en comparación con el resto de las obras recogidas: 85 frente a 2031 .

La Biblioteca de Andalucía supera, en número global de obras de Bellas Artes de tema andaluz, a la Biblioteca Nacional. Pero esta ventaja no la consigue en todos los años del período estudiado, ni en todas las variedades de documentos recopilados.

Las bibliotecas centrales autonómicas cumplen una misión importante al disponer de todo lo publicado en el territorio autonómico (así bastantes usuarios utilizan los fondos sin efectuar largos desplazamientos) y sobre todo al aumentar la cobertura, pues recogen mayor cantidad y variedad de documentos (que no llegan a la Biblioteca $\mathrm{Na}$ cional o que ésta no los tiene en cuenta). En este sentido -si los medios económicos y legislativos lo permitieran- me parecería muy útil que la colección que guarda una biblioteca de estas características estuviera también en cada una de las provincias, sobre todo en comunidades tan extensas como la andaluza. Así se evitaría, a la hora de diseñar la estructura bibliotecaria autonómica, la sustitución de un centralismo (el de Madrid) por el de otra ciudad cualquiera.

La duplicación de esfuerzos que supone confeccionar una bibliografía nacional y otra bibliografía autonómica, ambas basadas en el Depósito Legal y que tanto cuestionan los seguidores del control bibliográfico español, es una realidad, según este estudio. Además he querido cuantificar la duplicación de obras existente en el campo temático que he seleccionado.

A través de la investigación realizada he podido observar algunos datos del cumplimiento del Depósito Legal: en la comparación entre los fondos depositados en la Biblioteca de Andalucía y los de la Biblioteca Nacional he detectado diferencias en número de obras. Pueden deberse, en unos casos, a que en la Biblioteca Nacional el periodo de tiempo existente entre la recepción de las obras y su puesta a disposición de los usuarios es mayor; en otros casos, a fallos en la recepción y/o distribución de las publicaciones ingresadas por el Depósito Legal. Según los especialistas, el depósito de libros, folletos y publicaciones periódicas -lo que abarca nuestro estudio- es bastante correcto. La problemática actual parece estar centrada en la necesidad de mejorar la legislación vigente para que incluya, entre los documentos que manda recoger, la literatura gris junto a los nuevos soportes y formas de comunicación vinculados a las nuevas tecnologías. Además de este problema de la cobertura documental se citan otros como: la falta de medios suficientes para poner a disposición de los usuarios, de forma rápida y adecuada, los fondos provenientes del Depósito Legal y el incumplimiento de éste por parte de muchos impresores, sobre todo en los materiales que no son folletos, libros, ni publicaciones periódicas. 
El Depósito Legal constituye, hoy día, la fuente más eficaz para localizar la mayor parte de lo que se publica en nuestro país, a pesar de las mejoras necesarias y de los errores que se detectan.

\section{Bibliografía}

1. CORDÓN GARCfA, J. A. El Control Bibliográfico Nacional en el contexto del Control Bibliográfico Regional: Andalucía. Boletín de la Asociación Andaluza de Bibliotecarios, 1996, año 12, n. $^{\circ} 44$, septiembre, pp. 63-81.

2. CARRIÓN GÚTIEZ, M. Bibliografía nacional y Autonomías. Boletín de la ANABAD, 1985, XXXV, n. ${ }^{\circ}$ 2-3, pp. 315-322.

3. TORRES RAMIREZ, I. de. De nuevo sobre la bibliografía general nacional periódica en España: historia y presente. Boletín de la Asociación Andaluza de Bibliotecarios, 1991, año 7, n. ${ }^{\circ} 24$, septiembre, pp. 5-23.

4. CORDON GARCIA, J. A. El registro de la memoria: las bibliografías nacionales y el Depósito Legal. Gijón: TREA, 1997.

5. GIRÓN GARCfA, A. El control bibliográfico en España. En: Primer Congreso de Bibliografía Asturiana, Oviedo, 11-14 de abril de 1989, Oviedo: Biblioteca de Asturias, 1991, pp. 35-54.

6. FUENTES ROMERO, J. J. Folletos y publicaciones menores en el Depósito Legal: las bibliotecas y la región. Madrid: Comisarfa Nacional de Bibliotecas, 1977. 